\title{
Elastic plastic deformation of TC6 titanium alloy analyzed by in-situ synchrotron based X-ray diffraction and microstructure based finite element modeling
}

Ran Shi ${ }^{\mathrm{a}, \mathrm{b}}$, Zhihua Nie ${ }^{\mathrm{b}}$, Qunbo Fan ${ }^{\mathrm{a}, \mathrm{b}, *}$, Guoju Li ${ }^{\mathrm{a}, \mathrm{b}}$

${ }^{a}$ National Key Laboratory of Science and Technology on Materials Under Shock and Impact, Beijing 100081, China

${ }^{b}$ School of Materials Science and Engineering, Beijing Institute of Technology, Beijing 100081, China

* Corresponding author: Email: fanqunbo@ bit.edu.cn, Tel: 8610-68911144-863, Fax:

8610-68911144-866

\section{Abstract}

The phase properties and distribution of multi-phase materials not only produce heterogeneous stress distributions between phases, but also stress gradients inside grains. However, due to limitations of stress/strain partitioning at the submicron scale, mechanical property characterizations of such alloys are typically based on evaluating averaged responses, e.g. macroscopic stress-strain curves. Here, the elastic plastic deformation of $\alpha / \beta$ dual phase TC6 titanium alloy is investigated in depth on the microstructural level by a two dimensional microstructure based finite element method (FEM). In-situ synchrotron based X-ray diffraction was also used to characterize the lattice strain evolutions during deformation. The microscopic stress and strain evolutions 
were successfully tracked by FEM during the elastic plastic transition process. The $\alpha$ phase bears a relatively high stress compared to the $\beta$ phase during the elastic deformation stage. In the elastic to plastic deformation stage, a stress inversion phenomenon was observed in the microstructure by path tracing to elucidate the stress transfer process in detail. In the plastic deformation stage, strong plastic strain concentration occurs in the $\alpha$ phase near phase boundaries. The current local strains and stresses of $\alpha$ and $\beta$ phases are not synchronous with the applied macroscopic true strains and the corresponding constitutive stresses. Moreover, different current local strain rates produce obvious stress gradients inside the $\alpha$ grains.

\section{Keywords:}

Metals and alloys;

Mechanical properties;

Microstructure;

Computer simulations;

Synchrotron radiation.

\section{Introduction}

In recent years, significant attention has been directed towards the TC6 (Ti-6Al-2.5Mo-1.5Cr-0.5Fe-0.3Si) titanium alloy $[1,2]$. Its high relative strength, good ductility, toughness, high strength-to-weight ratio, and excellent corrosion resistance make it a versatile material that is used in many fields, such as the automotive and 
aerospace industries. Particularly, the mechanical properties of titanium alloys are important performance criteria for industrial applications [3].

The microstructure is one important factor that controls the mechanical properties of the alloys. Ghosh and Anahid developed an anisotropic yield function with a direct connection to the morphology and crystalline structure of single-phase $\alpha$-Ti-6Al [4]. Based on this function, they established a macroscopic probabilistic crack nucleation model of polycrystalline single phase $\alpha$-Ti alloys [5]. Such model was expected to capture important deformation characteristics of Ti-based alloys, which were pressure insensitivity, anisotropy, and tension-compression asymmetry. While in the practical applications, titanium alloys usually have multi-phase microstructures. Ti-6Al-4V alloy which consists of $\alpha+\beta$ phases has been systematically studied to investigate the effects of strain rates and temperatures, multiaxial and non-proportional loading responses, and deformation induced anisotropic responses [6-8]. Smith et al. investigated the cycle deformation behavior of three $\alpha-\beta$ titanium alloys with different microstructures: a microstructure of complete $\alpha+\beta$ colony grains, a microstructure of fine bi-modal phases, and a Widmanstatten microstructure [9]. Their studies are very helpful for tailoring microstructures to reach target property sets. As a dual-phase titanium alloy, the original forging TC6 has a microstructure that contains a mixture of equiaxial primary $\alpha$ grains and precipitated secondary $\alpha$ grains in $\beta$ matrix. This mixture combines the strength and ductility of the $\alpha$ and $\beta$ phases, respectively. During deformation, the $\alpha$ and $\beta$ phases, which have contrasting mechanical characteristics, give rise to heterogeneous stress 
partitioning and inhomogeneous stress/strain distributions. Thus, to understand the underlying mechanism causing the strong coupling between mechanical properties and microstructure, an analysis of elastic plastic transition process is crucial.

The micromechanical behavior, especially the stress/strain partitioning between phases during the elastic plastic transition process, has been widely studied experimentally by in-situ X-ray diffraction [10-13], in-situ neutron diffraction [14, 15], and digital image correlation (DIC) [16]. Among those techniques, in-situ high energy X-ray diffraction (HEXRD) shows its advantages. By using the HEXRD, Jia et al. investigated the stress partitioning of soft and hard phases in advanced high-strength steels [10]. Choi et al. determined the mechanical properties of the constituent phases in transformation-induced plasticity (TRIP) steels and estimated the phase volume fractions $[11,12]$. For titanium alloys, different crystal structures of a hexagonal close-packed (hcp) $\alpha$ phase and a body-centered cubic (bcc) $\beta$ phase make it complex to determine the mechanical properties of each phase. Stapleton et al. characterized the evolution of intergranular and interphase microstrain in Ti-6Al-4V [13]. There have been few reports on how microstructural features affect the accommodation of stress and strain during elastic plastic deformation in titanium alloys.

To combine the micromechanical behavior with the microstructure of materials, experimental techniques and numerical simulation methods have been integrated and improved. Among those numerical simulation methods, finite element method (FEM) has been developed to be a powerful tool. The particle orientation-induced changes in 
stress-strain behavior of SiC particle reinforced Al matrix composites were modeled using FEM [17]. FEM modeling quantitatively predicted the strength and ductility of the dual phase steels and the failure modes with different martensite volume fractions [18]. The crystallographic and morphological microstructural features can be reconstructed using mathematically adaptive algorithms or 3-D CT and electron backscattered diffraction (EBSD) techniques [19-21]. Therefore, microstructure based FEM must be extended to realistic microstructures to take advantage of each phase's constitutive relation by accounting for each phase's microscale morphology. There are, however, only few recent works $[22,23]$ that integrate the simulated deformation induced microscopic strain or stress evolution with experimental analysis.

However, the complex real microstructures for multi-phase materials are still difficult to capture. For modeling convenience, appropriate simplifications are always performed on the microstructural models, especially for titanium alloys. By applying single crystal continuum constitutive equations at the grain and polycrystal scale, the crystal plasticity finite element (CPFE) modeling can effectively represent the micromechanical behavior of the alloys. The cyclic loading history of duplex Ti-6Al-4V was fitted by Zhang et al. using CPFE modeling [24], however, the grain sizes were assumed to be identical based on qualitative observation. Chan and Lee [25] verified the effects of deformation-induced constraint on high cycle fatigue in Ti-6Al-4V, whereas the lamellar $\alpha+\beta$ colonies were treated as units with the same constitutive properties of the experimental relevant constant of $\beta$-Ti-Mn. Li et al. [26] synchronously considered the 
dynamic recrystallization and thermomechanical behavior of wrought two-phase titanium alloys, which consist of the primary $\alpha$, the lamellar $\alpha$, and the matrix $\beta$, but the lamellar $\alpha$ in the microstructure was not taken into account. Thus, accounting for the significantly differential properties and morphology distributions of the $\alpha$ and $\beta$ phases is an essential issue for modeling.

In this study, the elastic plastic deformation process of TC6 titanium alloy were investigated by numerical simulation and experiment. Stress/strain partitioning and transition processes between $\alpha$ and $\beta$ phases were studied from the aspect of mechanical properties and also on the microstructural level. We used in-situ HEXRD to capture in-depth information about the lattice strain evolutions during deformation. This information is also essential for obtaining the constitutive relations of each phase as input for FEM. The FEM model was constructed based on a realistic microstructure obtained by scanning electron microscopy (SEM) images, which detailed the phase morphology, size, and boundary distribution. The evolutions of microscopic stress and strain were analyzed by employing stress/strain contours and path tracing. Strain partitioning between $\alpha$ and $\beta$ phases in the local microstructure was investigated to explain differences between the current local stresses and the stresses in the constitutive relation corresponding to the applied macroscopic true strain. Local strain rate evolutions were then applied to interpret the stress gradient inside the $\alpha$ grains and the preferential regions for initiating failure were predicted.

\section{Experimental description}


In-situ synchrotron HEXRD experiments were performed in the 11-ID-C beamline at the Advanced Photon Source (APS), Argonne National Laboratory. A monochromatic X-ray beam with energy of $111 \mathrm{keV}$ (with wavelength of $0.11165 \AA$ ) was used to map the lattice strain distributions of the specimen under uniaxial tensile loading. The experimental set-up is schematically shown in Fig. 1. The dog-bone-shaped sheet specimen of forged TC6 titanium alloy with gauge section dimensions of $3.175 \mathrm{~mm} \times$ $10.16 \mathrm{~mm} \times 1 \mathrm{~mm}$ was prepared by mechanically grinding and polishing. At each loading level, diffraction patterns were collected by a two dimensional (2D) detector placed at approximately $1.5 \mathrm{~m}$ behind the sample, with sampling times of $\sim 1 \mathrm{~s} . \mathrm{CeO}_{2}$ powder was used as standard sample for calibrating the tilt angles and sample-to-detector distance.

The lattice strain distribution and evolution of different phases in the titanium alloy sheet can be investigated by tracing the change in lattice spacing with increasing load. The interplanar spacing of the $h k l$ plane, $d_{h k l}$, is obtained by Bragg's law, $d_{h k l}=\frac{\lambda}{2 \sin \theta_{h k l}}$

where $2 \theta_{h k l}$ is the diffraction angle of a Debye cone. The Bragg angle $\theta_{h k l}$ of an $h k l$ plane is determined by fitting an individual peak or overlapped peaks in the diffraction spectra by employing two Gaussian functions with different peak widths. The detailed procedures were demonstrated by Jia et al. [10, 27] and Stapleton et al. [13].

Measured HEXRD lattice strains in response to applied stress along the loading direction in the $\alpha$ and $\beta$ phases are shown in Fig. 2. During the initial stage, all the lattice 
strains respond linearly to the applied stress, which indicates that only elastic deformation occurs in both phases. Different slopes are found for different reflections, corresponding to their respective moduli. After linear fitting, the $\{110\}_{\beta}$ reflection, which has the lowest slope of all those observed in the $\beta$ phase, has a slope larger than any reflection in the $\alpha$ phase, indicating that the $\alpha$ phase has a higher Young's modulus than the $\beta$ phase. Therefore, significant load partitioning is expected prior to the $\alpha$ phase during the elastic

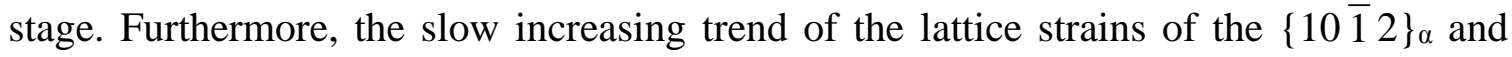
$\{0002\}_{\alpha}$ reflections at $910 \mathrm{MPa}$ indicates the start of a plastic flow in the $\alpha$ phase. The lattice strains of the $\{10 \overline{1} 2\}_{\alpha}$ and $\{0002\}_{\alpha}$ reflections then reduce slightly at $952 \mathrm{MPa}$. Meanwhile, all the reflections of the $\beta$ phase exhibit larger elastic lattice strains. This trend suggests that the $\beta$ phase is still subjected to increasing stress at this stage of deformation when there is a stress relaxation in the $\alpha$ phase. At approximately $964 \mathrm{MPa}$, the lattice strains of $\{10 \overline{1} 1\}_{\alpha},\{10 \overline{1} 2\}_{\alpha}$ and $\{0002\}_{\alpha}$ reflections decrease rapidly except for $\{10 \overline{1} 0\}_{\alpha}$. This shows that a large proportion of the applied load is transferred to other, un-yielded, $\alpha$ and $\beta$ grains. When the load increases further, the stress partitioning continues to vary among reflections and phases, which is resultant of the plastic anisotropy arising from grain-to-grain interactions.

Pole figures were obtained for the $\alpha$ and $\beta$ phases by EBSD experiment using Channel 5 software (Fig. 3). In $\{0002\}_{\alpha}$ pole figure, the main orientation component is located at about $50^{\circ}$ to the loading axes, and the main orientation component in $\{100\}_{\beta}$ is inclined at about $70^{\circ}$ to the loading axes. The maximum intensity is 2.75 and 3.06 for 
planes of $\alpha$ and $\beta$ phases, respectively. The uniaxial tensile specimen was tested along the normal axis. The orientation and volume fraction of 2500 grains in each phase used for SC modeling were specified by a set of discrete Euler angles weighted to match the EBSD measured texture.

\section{Finite element numerical simulations}

\subsection{Creating the finite element grid model}

The microstructure of original forged TC6 titanium alloy obtained by SEM on the longitudinal surface is displayed in Fig. 4(a). It consists of equiaxial primary $\alpha$ grains and precipitated secondary $\alpha$ grains in $\beta$ matrix. Primary $\alpha$ grains are $\sim 13 \mu \mathrm{m}$ and secondary $\alpha$ grains $\sim 3 \mu \mathrm{m}$. Elastic plastic finite element analysis is performed based on this realistic microstructure. By converting the image to a gray scale digital image composed of pixels, one phase can be extracted from the other by selecting a thresholding gray scale value. Similarly, based on the same principle, metallographic analysis software can identify the volume fraction $(V)$ of each phase: $V(\alpha) \approx 80 \%$ and $V(\beta) \approx 20 \%$. With one pixel corresponding to one finite element, a collection of pixels can be transferred to a collection of finite elements $[28,29]$. In this way, a finite element grid model $(143 \mu \mathrm{m} \times$ $162 \mu \mathrm{m}$ ) containing 234,3254 -node square elements (455 pixels $\times 515$ pixels) is shown in Fig. 4 (b).

\subsection{External load, boundary conditions, and material properties}


Numerical simulations are performed using LS-DYNA code to model uniaxial tensile loading in a quasi-static state. Right and left boundaries are fixed at equal displacements in the $x$ direction, while they can freely move in the $y$ direction. The top boundary is subjected to quasi-static normal stress that is defined by a constant velocity in the tensile direction to ensure a constant strain rate of $\dot{\varepsilon}=2.78 \times 10^{-5} \mathrm{~s}^{-1}$, and the bottom boundary is fixed. The macroscopic engineering stress $(\sigma)$ is defined by dividing the reaction force by the initial area (initial top boundary length $\times$ default $2 \mathrm{D}$ element thickness), and the macroscopic engineering strain $(\varepsilon)$ is obtained by dividing the displacement of the top boundary by the initial length of the model in the $y$ direction. The true stress-strain curve is obtained from the engineering stress-strain curve using the following equations:

$\varepsilon_{T}=\ln (1+\varepsilon)$

$\sigma_{T}=\sigma(1+\varepsilon)$

The simulated macroscopic true stress-strain response, as shown in Fig. 5, is in good agreement with the macroscopic true stress-strain curve obtained by in-situ HEXRD experiment. The respective stress-strain curves for each individual phase obtained from the self-consistent (SC) model are also shown which form the input for FEM. The elastic plastic SC framework, first implemented by Hutchinson [30], has proven effective for predicting macroscopic stress-strain response [31, 32]. Adjusting the SC framework parameters (the single crystal elastic constants, and critical resolved shear stresses and hardening parameters), the constitutive relations for each phase in TC6 titanium alloy were obtained. The simulated macroscopic true stress-strain response lies in the middle of the stress-strain curves for the $\alpha$ and $\beta$ phases. The $\alpha$ phase has a larger Young's modulus 
than $\beta$ phase. Plastic deformation takes place preferentially in the $\alpha$ phase and subsequently in the $\beta$ phase. These trends agree with observations of lattice strain responses of diffraction reflections (see Section 2). When both phases yield, $\alpha$ phase hardens more rapidly than $\beta$ phase with increasing plastic deformation. In LS-DYNA software, the isotropic elasto-plastic material model (*MAT_PIECEWISE_LINEAR_PLASTICITY) is used to describe the constitutive relations for each phase. The model parameters such as the Young's modulus $\left(E_{\alpha}=116.6 \mathrm{GPa}\right.$ and $\left.E_{\beta}=103.4 \mathrm{GPa}\right)$ and initial yield stress $\left(\sigma_{y, \alpha}=878 \mathrm{MPa}\right.$ and $\left.\sigma_{y, \beta}=975 \mathrm{MPa}\right)$ can be obtained from the simulated stress-strain curves for the $\alpha$ and $\beta$ phases, and the effective stress versus effective plastic strain curve are assumed to be in the following equations, respectively:

$\sigma_{\alpha}=\sigma_{y, \alpha}+R_{\alpha} \varepsilon_{p}^{n_{\alpha}}$

$\sigma_{\beta}=\sigma_{y, \beta}+R_{\beta} \varepsilon_{p}^{n_{\beta}}$

where $R_{\alpha}=1208 \mathrm{MPa}$ and $R_{\beta}=318 \mathrm{MPa}$ represent the strength coefficients and $n_{\alpha}=0.6$ and $n_{\beta}=0.3$ represent the hardening exponent for the $\alpha$ and $\beta$ phases, respectively.

\section{Results and discussion}

\subsection{Elastic plastic deformation process on the microstructure level}

As demonstrated in Fig. 5, the whole elastic plastic deformation process can be divided into three stages according to the deformation behavior characterized by FEM: (I) the elastic deformation stage (macroscopic strain $\varepsilon_{T}<0.0075$ ), (II) the elastic to plastic deformation stage $(0.0075<\varepsilon T<0.01)$, and (III) the plastic deformation stage $(0.01<\varepsilon T)$. 
We selected four points, designated as A, B, C, and D in Fig. 5, to analyze these stages individually.

The plastic strain and Von Mises effective stress contours at A, B and C are shown in Fig. 6. Point A $(\varepsilon T=0.005)$ was chosen to be in the elastic deformation stage (Fig. 6(a)), and the plastic strain of the whole area remains at zero until plastic deformation begins in the $\alpha$ phase at $\mathrm{B}(\varepsilon T=0.0075)($ Fig. $6(b))$. When the $\beta$ phase also deforms plastically, $\mathrm{C}(\varepsilon T=0.01)$ is the end point of the elastic to plastic stage. Point D $\left(\varepsilon_{T}=0.05\right)$ is at the end of the simulation.

To capture the mechanical behaviors of different microstructural details, we propose a path tracing method, as shown in Fig. 7 . We chose a path in the transverse direction from the initial finite element grid model, and the effective stress distributions at different macroscopic true strains can be traced. Elements along the path can be classified according to their phase properties to analyze the local stress and strain partitioning between $\alpha$ and $\beta$ phases.

\subsubsection{Stage I: elastic deformation stage}

During the elastic deformation stage, $\alpha$ and $\beta$ phases both deform elastically. Figs. 6(a) and (b), and (d) and (e) compare the plastic strain and Von Mises effective stress distributions at points $\mathrm{A}$ and $\mathrm{B}$, respectively. The initial occurrence of plastic deformation and stress concentration in the $\alpha$ phase can be ascribed to its lower yield stress and higher stress partitioning during the elastic stage, as discussed in Section 2. 
The effective stress distributions at different macroscopic true strains can be traced from $\varepsilon_{T}=0.005$ to 0.0075 (from points A to B) along the selected path. In Fig. 8(a), path tracing shows that the stresses of $\alpha$ phase grains occur at the peaks while the stresses of $\beta$ phase grains occur at the troughs, which is consistent with the stress partitioning conclusions obtained from X-ray diffraction (Fig. 2) during the elastic deformation stage. Local stress concentration is evident in most positions near the phase boundaries as positions a, b, c, and d as marked in Fig. 8(a). The stress difference between position a and the grain center changes from $17 \mathrm{MPa}$ to $23 \mathrm{MPa}$ (from $\varepsilon_{T}=0.005$ to 0.0075 ). Fig. 8(b) shows the respective average effective stresses among $\alpha$ and $\beta$ phases along the predefined local path. Noticeably, for the dual phase alloy, the current local strain of each phase might not be synchronous with the applied macroscopic true strain of the sample. Thus the current local stresses differ from the stresses in the constitutive relation corresponding to the applied macroscopic true strain, as shown in Fig. 5. To observe such stress differences for each phase, the constitutive stress-strain curves are also illustrated in Fig. 8(b) as references. The $\alpha$ phase shows lower stress in the current local path than its counterpart in the constitutive relation. This is due to the local strain hysteresis during load initiation, which leads to smaller local strain than the macro loading strain, as shown in Fig. 8(c). For the $\beta$ phase, the stress of the constitutive relation should also be much higher for the same reason. However, higher stress is evident in the current local path for the $\beta$ phase. Such paradoxical phenomenon arises from the requirement of stress equilibrium and strain compatibility of the sample. The local stress and strain of $\beta$ phase 
are higher than expected to compensate for the gap between the local and the overall deformation, which is also confirmed in Fig. 8(c). In addition, the $\alpha$ phase dominates stress partitioning during elastic deformation stage due to its larger Young's modulus.

\subsubsection{Stage II : elastic to plastic deformation stage}

Comparing Figs. 6 (b) and (c), and (e) and (f), the plastic strain is higher in the $\alpha$ phase and the stress in the $\beta$ phase is much higher than in the $\alpha$ phase for this stage. As shown in Fig.9(a), effective stress distributions along the path as $\varepsilon T$ increases from 0.0075 to 0.01 (from point B to C) can be tracked explicitly on the microstructural level. The stress state of the $\alpha$ phase reduces, while the $\beta$ phase increases, a phenomenon known as stress inversion. In Fig.9(b), the low stress growth in $\alpha$ phase indicates that the accumulation of stress during elastic deformation stage triggers the plastic sliding of slip systems. The stress in the $\alpha$ phase then releases and transfers to the $\beta$ phase. Combining with Fig.9(c), the average effective strain of $\alpha$ phase along the current local path increases with the applied macroscopic true strain and catches up with $\beta$ phase when $\varepsilon T$ $\approx 0.00875$. When $\varepsilon_{T} \approx 0.00882$, the local path stress of $\beta$ phase surpasses that of $\alpha$ phase. This indicates that once the $\alpha$ phase yields and deforms plastically, more stress is borne by the $\beta$ phase, and stress partitioning and transfer are implemented by the partitioning and coordination of local strains of two phases. From there, the stress difference of the $\beta$ phase between the current local path and the constitutive relation tends to increase, While for the $\alpha$ phase, the stress difference reduces gradually. 


\subsubsection{Stage III: plastic deformation stage}

The effective stress distributions along the selected path as $\varepsilon T$ increases from 0.011 to 0.05 (point $\mathrm{C}$ to $\mathrm{D}$ ) are shown in Fig. 10(a). On the microstructural level, the $\beta$ phase bears a higher stress than the $\alpha$ phase, and there is no stress inversion. Lower stress occurs at the positions where local stress concentrated during elastic deformation stage (marked a, b, c, and d, and also shown in Fig. 8(a)). The maximum stress difference between position a and the grain center is $15.6 \mathrm{MPa}$ at $\varepsilon r=0.03$.

As shown in Fig. 10(b), after entering into the plastic deformation stage, the stress along the current local path of the $\beta$ phase gradually approaches its constitutive value, whereas for $\alpha$ phase, the stress difference between current local and constitutive relation tends to increase. To interpret this trend, Fig. 11 shows the contours of the Von Mises effective stress and plastic strain at point $\mathrm{D}\left(\varepsilon_{T}=0.05\right)$. The stress in the $\beta$ phase $(\sim 1150$ MPa) is much higher than the stress in the $\alpha$ phase ( 1000 MPa) (Fig. 11(a)). However, the plastic strain in the $\alpha$ phase is still larger than that in the $\beta$ phase, as shown in Fig. 11(b). The local amplifications (Figs. 11 (b)-1 and (b)-2) show the strong plastic strain concentration in the $\alpha$ phase near phase boundaries, which makes the $\alpha$ phase local strain remain larger than the applied macroscopic strain. Accordingly, the stress along the current local path of the $\alpha$ phase maintains a relatively steady extending trend comparing with the constitutive relation. From the simulation, we know that strain concentration initiates from several points and then extends to interior regions gradually. The average 
effective strain evolutions of the $\alpha$ and $\beta$ phases in the current local path are shown in Fig. 10(c), where the $\alpha$ phase shows significantly more deformation than the $\beta$ phase.

\subsection{Stress gradient inside $\alpha$ grains}

In the above analyses, stress gradient inside $\alpha$ grains can be found in both the elastic and plastic deformation stages. To probe the underlying mechanism, we compared effective strain rates of three positions in the $\alpha$ grain along the selected path from a position near the phase boundary to the grain center (positions 1,2,3), as shown in Fig. 12. During elastic deformation, the effective strain rate of position 1 is $\sim 2.5 \times 10^{-5} \mathrm{~s}^{-1}$, and $\sim 2.35 \times 10^{-5} \mathrm{~s}^{-1}$ for positions 2 and 3 . Position 1 has a relatively higher strain rate, so the strain leads that of other positions and the stress is more inclined to congregate near the phase boundary. Position 1 then enters primarily into the plastic deformation stage, and the strain rate increases to $3.2 \times 10^{-5} \mathrm{~s}^{-1}$. Subsequently, stress release starts when positions 2 and 3 reach their critical yield stresses and also enter plastic deformation. The strain rates of positions 1 and 2 are influenced not only by the nearby $\beta$ phase but also the stress transfer from the grain center position, which make them drop sharply with different levels. From their distances to grain center, the strain rates drop to $0.97 \times 10^{-5} \mathrm{~s}^{-1}$ and $2.6 \times 10^{-5} \mathrm{~s}^{-1}$, respectively. Therefore, the significant strain rate gradient inside $\alpha$ grain is the cause of the stress gradient. During plastic deformation, the effective strain rate evolutions of these positions first converge then separate with the consequence that position 1 has the highest value. This is very significant, as it means that stress and strain 
will finally concentrate in the $\alpha$ phase particularly near the phase boundary. Thus, failure will initiate preferentially at these regions.

\section{Conclusions}

This study investigated the elastic plastic transition process of a TC6 titanium alloy. Realistic microstructure based FEM was applied to the transition process and divided it into three typical stages: elastic deformation, elastic to plastic deformation, and plastic deformation. Good agreement was achieved between the FEM simulation and HEXRD for the macro stress-strain curve.

Initial plastic deformation occurred in the $\alpha$ phase because of its lower yield stress and higher stress partitioning during the elastic deformation stage. Higher stress was recorded in the current local path for $\beta$ phase than in the constitutive relation corresponding to the applied macroscopic true strain. To satisfy the requirement of stress equilibrium and strain compatibility, current local strain in $\beta$ phase lead the applied macroscopic true strain. Notably, during the elastic to plastic deformation stage, the stress inversion phenomenon and stress transfer process were successfully captured on the microstructural level and implemented by the partitioning and coordination of the local strains between phases. During the plastic deformation stage, $\beta$ phase showed higher stress than $\alpha$ phase. Strong plastic strain concentration in the $\alpha$ phase near phase boundaries was observed, and, hence, stress difference between current local and constitutive relation tended to increase, and $\alpha$ phase accumulated more deformation than $\beta$ phase at the end of the model. 
Stress gradients inside $\alpha$ grains were found in both elastic and plastic deformation stages. This can be ascribed to the strain rate gradient from phase boundary to grain center. At the end of the simulation, the highest effective strain rates were found at positions near phase boundaries. Stress and strain concentration in the $\alpha$ phase especially near the phase boundary will initiate failure there preferentially.

\section{Acknowledgements}

We gratefully acknowledge the financial support of the program for New Century Excellent Talents in University (NCET-12-0051), and funding support from project 51571031 of the Chinese National Natural Science Foundation. Use of the Advanced Photon Source was supported by the US Department of Energy, Office of Science Laboratory, Office of Basic Energy Sciences, under contract DE-AC02-06CH11357. The assistance of Dr. Yan-Dong Wang of the University of Science and Technology Beijing with the self-consistent modeling aspects is greatly appreciated.

\section{References}

[1] M.Q. Li, A.M. Xiong, S.H. Cheng, W.C. Huang, Y.C. Li, H. Lin, Effects of process parameters on the microstructure during the hot compression of a TC6 titanium alloy, Rare Metals. 23 (2004) 263-268.

[2] C.H. Pei, Q.B. Fan, H.N. Cai, J.C. Li, High temperature deformation behavior of the TC6 titanium alloy under the uniform DC electric field, J. Alloys Compd. 489 (2010) 401-407.

[3] G. Lütjering, J.C. Williams, Titanium, Springer, New York, 2003. 
[4] S. Ghosh, M. Anahid, Homogenized constitutive and fatigue nucleation models from crystal plasticity FE simulations of Ti alloys, Part 1: Macroscopic anisotropic yield function, Int. J. Plast. 47 (2013) 182-201.

[5] M. Anahid, S. Ghosh, Homogenized constitutive and fatigue nucleation models from crystal plasticity FE simulations of Ti alloys, Part 2: Macroscopic probabilistic crack nucleation model, Int. J. Plast. 48 (2013) 111-124.

[6] A.S. Khan, Y.S. Suh, R. Kazmi, Quasi-static and dynamic loading responses and constitutive modeling of titanium alloys, Int. J. Plast. 20 (2004) 2233-2248.

[7] A.S. Khan, R. Kazmi, B. Farrokh, Multiaxial and non-proportional loading responses, anisotropy and modeling of Ti-6Al-4V titanium alloy over wide ranges of strain rates and temperatures, Int. J. Plast. 23 (2007) 931-950.

[8] A.S. Khan, S.J. Yu, Deformation induced anisotropic responses of Ti-6Al-4V alloy. Part I: Experiments, Int. J. Plast. 38 (2012) 1-13.

[9] B.D. Smith, D. Shih, D.L. McDowell, Cyclic Plasticity Experiments and Polycrystal Plasticity Modeling of Three Distinct Ti Alloy Microstructures, Int. J. Plast. in press.

[10] N. Jia, Z.H. Cong, X. Sun, S. Cheng, Z.H. Nie, Y. Ren, P.K. Liaw, Y.D. Wang, An in situ high-energy X-ray diffraction study of micromechanical behavior of multiple phases in advanced high-strength steels, Acta Mater. 57 (2009) 3965-3977.

[11] K.S. Choi, W.N. Liu, X. Sun, M.A. Khaleel, Y. Ren, and Y.D. Wang, Advanced Micromechanical Model for Transformation-Induced Plasticity Steels with Application of 
In-Situ High-Energy X-Ray Diffraction Method, Metall. Mater. Trans. A. 39A (2008) 3089-3096.

[12] K.S. Choi, W.N. Liu, X. Sun, M.A. Khaleel, Microstructure-based constitutive modeling of TRIP steel: Prediction of ductility and failure modes under different loading conditions, Acta Mater. 57 (2009) 2592-2604.

[13] A.M. Stapleton, S.L. Raghunathan, I. Bantounas, H.J. Stone, T.C. Lindley, D. Dye, Evolution of lattice strain in Ti-6Al-4V during tensile loading at room temperature, Acta Mater. 56 (2008) 6186-6196.

[14] S. Harjo, Y. Tomota, P. Lukáš, D. Neov, M. Vrána, P. Mikula, M. Ono, In situ neutron diffraction study of $\alpha-\gamma \mathrm{Fe}-\mathrm{Cr}-\mathrm{Ni}$ alloys under tensile deformation, Acta Mater. 49 (2001) 2471-2479.

[15] X.P. Liu, R.L. Peng, M. Hofmann, S. Johansson, Y.D. Wang, In-situ neutron diffraction studies of micromechanical behavior in a friction stir welded AA7475-T761, Metall. Mater. Trans. A. 42 (2011) 89-94.

[16] C.C. Tasan, J.P.M. Hoefnagels, M. Diehl, D. Yan, F. Roters, D. Raabe, Strain localization and damage in dual phase steels investigated by coupled in-situ deformation experiments and crystal plasticity simulations, Int. J. Plast. 63 (2014) 198-210.

[17] V.V. Ganesh, N. Chawla, Effect of particle orientation anisotropy on the tensile behavior of metal matrix composites: experiments and microstructure-based simulation, Mater. Sci. Eng. A. 391 (2005) 342-353. 
[18] M. Marvi-Mashhadi, M. Mazinani, A. Rezaee-Bazzaz, FEM modeling of the flow curves and failure modes of dual phase steels with different martensite volume fractions using actual microstructure as the representative volume, Comput. Mater. Sci. 65 (2012) 197-202.

[19] M.A.S. Qidwai, A.C. Lewis, A.B. Geltmacher, Using image-based computational modeling to study microstructure-yield correlations in metals, Acta Mater. 57(2009) 4233-4247.

[20] J.F. Ramírez, M. Cardona, J.A. Velez, I. Mariaka, J.A. Isaza, E. Mendoza, S. Betancourt, P. Fernández-Morales, Numerical modeling and simulation of uniaxial compression of aluminum foams using fem and 3d-ct images, Procedia Materials Science. 4 (2014) 227-231.

[21] N. Vajragupta, P. Wechsuwanmanee, J. Lian, M. Sharaf, S. Münstermann, A. Ma, A. Hartmaier, W. Bleck, The modeling scheme to evaluate the influence of microstructure features on microcrack formation of DP-steel: the artificial microstructure model and its application to predict the strain hardening behavior, Comput. Mater. Sci. 94(2014) 198-213.

[22] G. Martin, C.W. Sinclair, R.A. Lebensohn, Microscale plastic strain heterogeneity in slip dominated deformation of magnesium alloy containing rare earth. Mater. Sci. Eng. A. 603(2014) 37-51. 
[23] C.C. Tasan, M. Diehl, D. Yan, C. Zambaldi, P. Shanthraj, F. Roters, D. Raabe, Integrated experimental-simulation analysis of stress and strain partitioning in multiphase alloys, Acta Mater. 81 (2014) 386-400.

[24] M. Zhang, J. Zhang, D.L. McDowell, Microstructure-based crystal plasticity modeling of cyclic deformation of Ti-6Al-4V, Int. J. Plast. 23 (2007) 1328-1348.

[25] K.S. Chan, Y.D. Lee, Effects of Deformation-Induced Constraint on High-Cycle Fatigue in Ti Alloys with a Duplex Microstructure, Metall. Mater. Trans. A. 39 (2008) 1665-1675.

[26] H.W. Li, C. Wu, H. Yang, Crystal plasticity modeling of the dynamic recrystallization of two-phase titanium alloys during isothermal processing, Int. J. Plast. $51(2013) 271-291$.

[27] N. Jia, R.L. Peng, Y.D. Wang, S. Johansson, P.K. Liaw, Micromechanical behavior and texture evolution of duplex stainless steel studied by neutron diffraction and self-consistent modeling, Acta Mater. 56 (2008) 782-793.

[28] W. Shen, Q.B. Fan, F.C. Wang, Z. Ma, Modeling of micro-crack growth during thermal shock based on microstructural images of thermal barrier coatings, Comput. Mater. Sci. 46 (2009) 600-602.

[29] W. Shen, Q.B. Fan, F.C. Wang, Z. Ma, X.W. Yang, Finite element simulation of tensile bond strength of atmospheric plasma spraying thermal barrier coatings, Surf. Coat. Tech. 205 (2011) 2964-2969. 
[30] J.W. Hutchinson, Elastic-plastic behaviour of polycrystalline metals and composites, Proceedings of the Royal Society A Mathematical Physical \& Engineering Sciences. 319 (1970) 247-272.

[31] M.R. Daymond, H.G. Priesmeyer, Elastoplastic deformation of ferritic steel and cementite studied by neutron diffraction and self-consistent modelling, Acta Mater. 50(2002) 1613-1626.

[32] M.R. Daymond, C.N. Tomé, M.A.M. Bourke, Measured and predicted intergranular strains in textured austenitic steel, Acta Mater. 48(2000) 553-564. 


\section{Figure captions}

Fig. 1. Schematic of the experimental set-up for in-situ loading studies by HEXRD.

Fig. 2. Response of measured HEXRD lattice strains to the applied stress for different reflections along the loading direction for: (a) $\alpha$ phase and (b) $\beta$ phase.

Fig. 3. Texture pole figures obtained by EBSD for: (a) the $\{0002\},\{11 \overline{2} 0\}$ and $\{10 \overline{1} 0\}$ $\alpha$ poles and (b) the $\{100\},\{110\}$ and $\{111\} \beta$ poles.

Fig. 4. (a) Microstructure of TC6 obtained by SEM; (b) the corresponding finite element grid model.

Fig. 5. FEM and experimental macroscopic true stress-strain responses under tensile loading, and the respective stress-strain curves for each individual phase obtained by SC model as the input for FEM.

Fig. 6. The plastic strain and Von Mises effective stress contours at points $\mathrm{A}\left(\varepsilon_{T}=0.005\right)$, $\mathrm{B}\left(\varepsilon_{T}=0.0075\right)$, and $\mathrm{C}\left(\varepsilon_{T}=0.01\right)$.

Fig. 7. The selected path from the initial finite element grid model in the transverse direction.

Fig. 8. (a) Effective stress distribution along the selected path as $\varepsilon_{T}$ increases from 0.005 to 0.0075 (point $\mathrm{A}$ to $\mathrm{B}$ ); (b) average effective stress partitioning among $\alpha$ and $\beta$ phases in the current local path and the effective stresses in the constitutive relations; (c) average effective strain partitioning in the current local path.

Fig. 9. (a) Effective stress distribution along the selected path as $\varepsilon_{T}$ increases from 0.0075 to 0.01 (point $\mathrm{B}$ to $\mathrm{C}$ ); (b) average effective stress partitioning among $\alpha$ and $\beta$ phases in the current local path and the effective stresses in the constitutive relations; (c) average effective strain partitioning in the current local path.

Fig. 10. (a) Effective stress distribution along the selected path as $\varepsilon_{T}$ increases from 0.01 to 0.05 (point $\mathrm{C}$ to $\mathrm{D}$ ); (b) average effective stress partitioning among $\alpha$ and $\beta$ phases in the current local path and effective stresses in the constitutive relations; (c) average effective strain partitioning in the current local path.

Fig. 11. Contours of (a) the Von Mises effective stress and (b) plastic strain at point D $\left(\varepsilon_{T}\right.$ $=0.05)$.

Fig.12. Effective strain rate evolutions of position 1 (near phase boundary), 2, and 3 (at grain center) in the $\alpha$ grain along the selected path. 


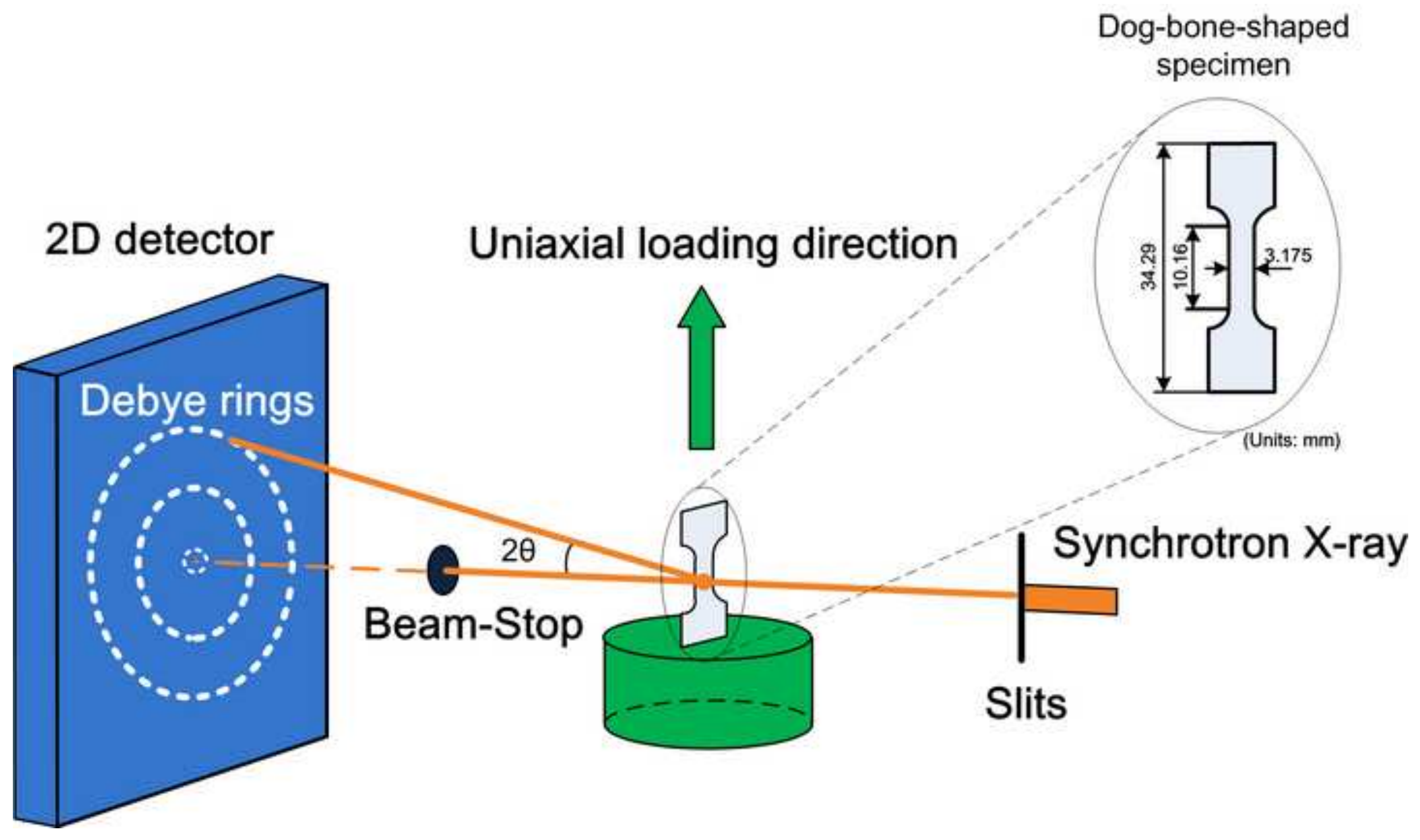



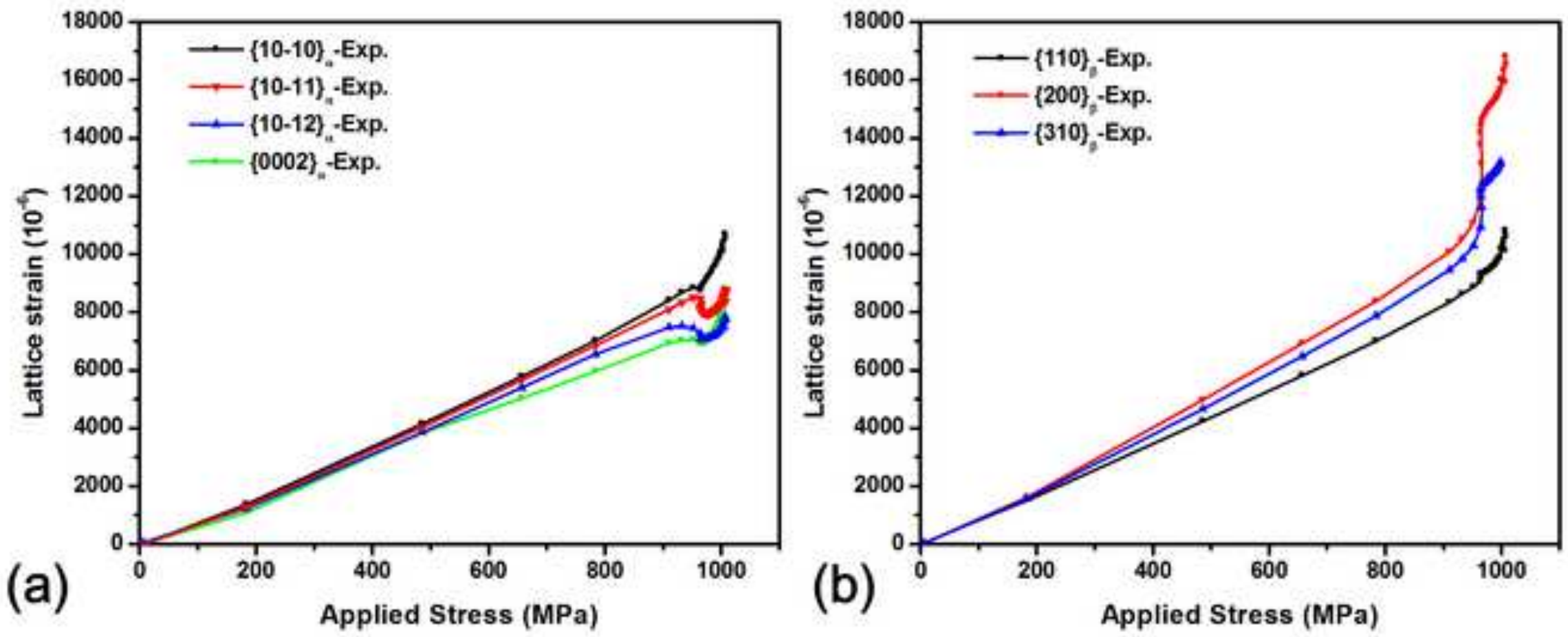

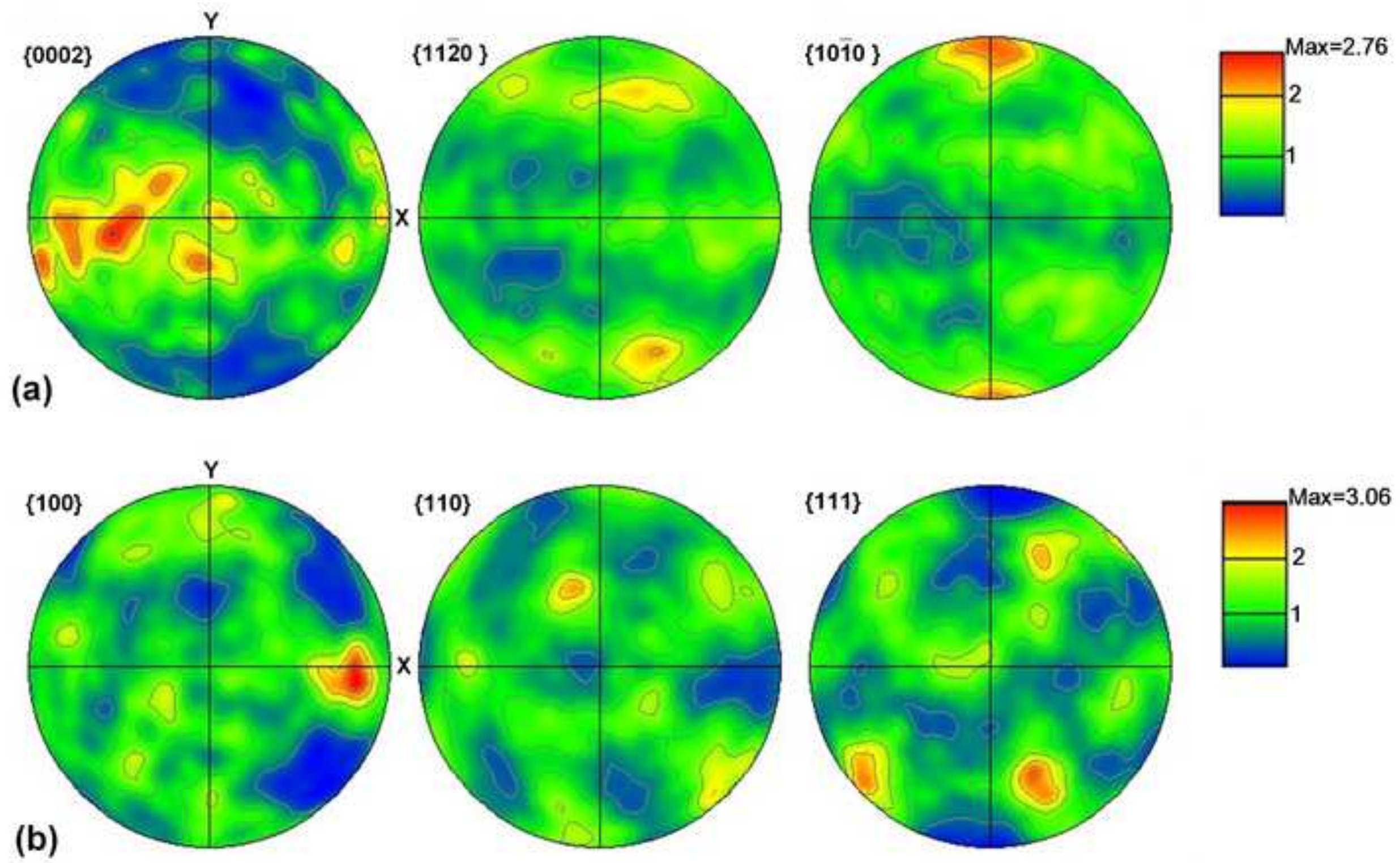

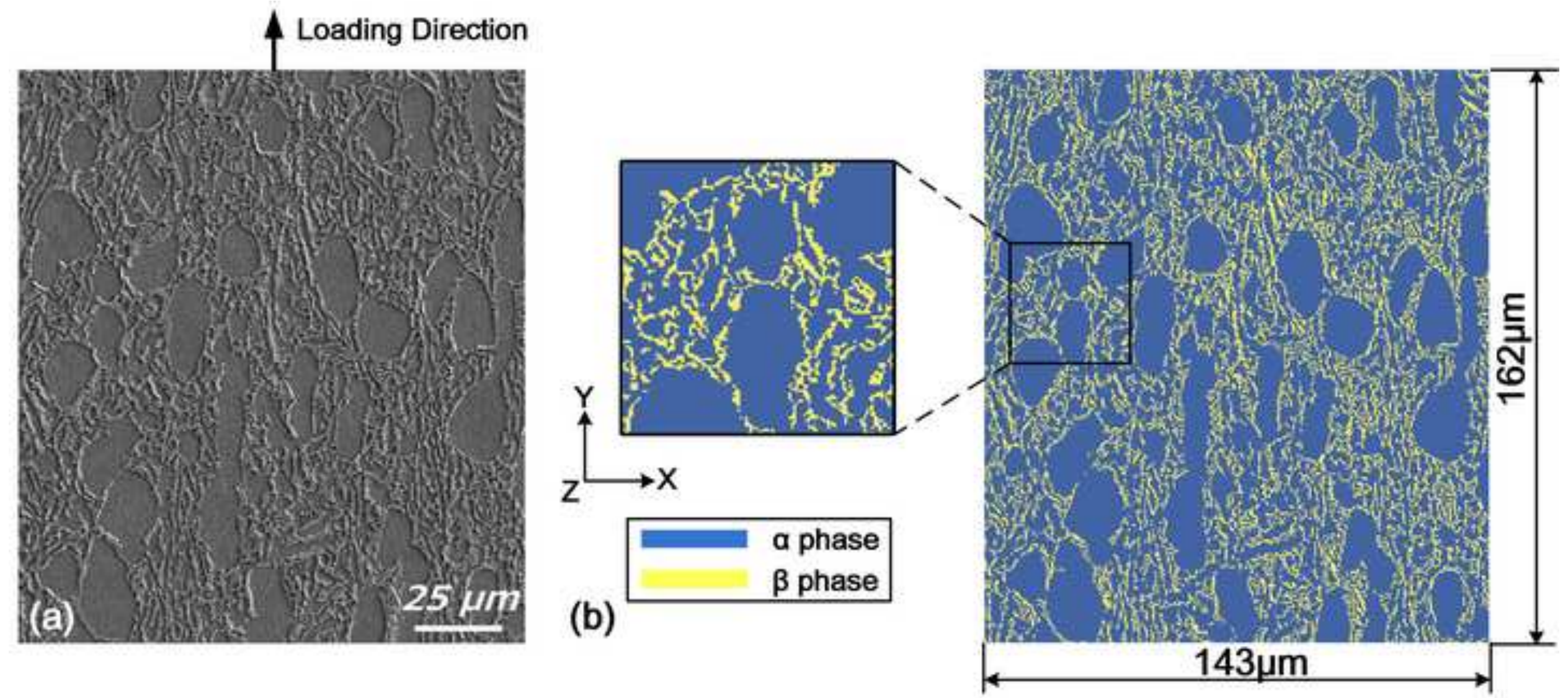


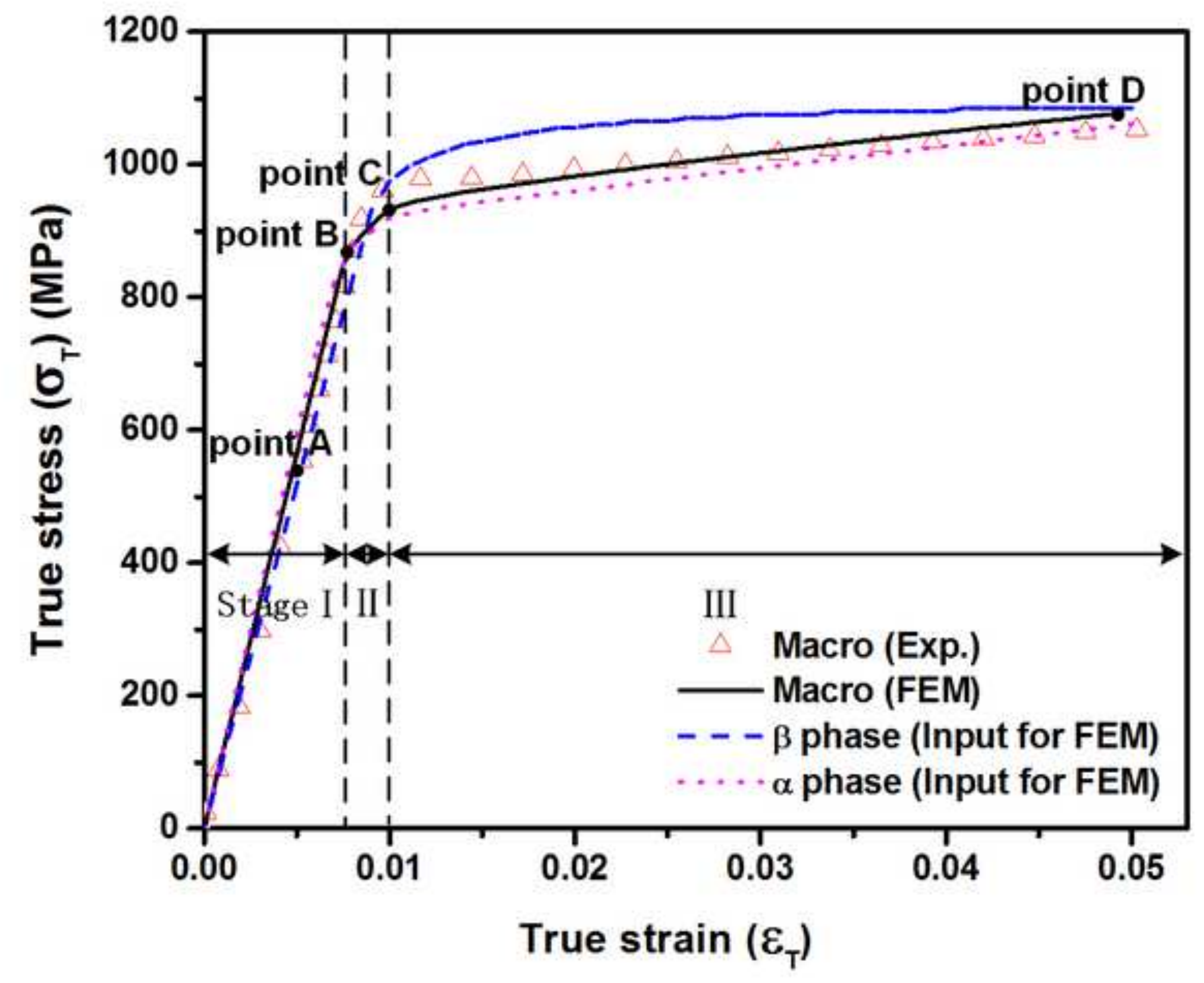




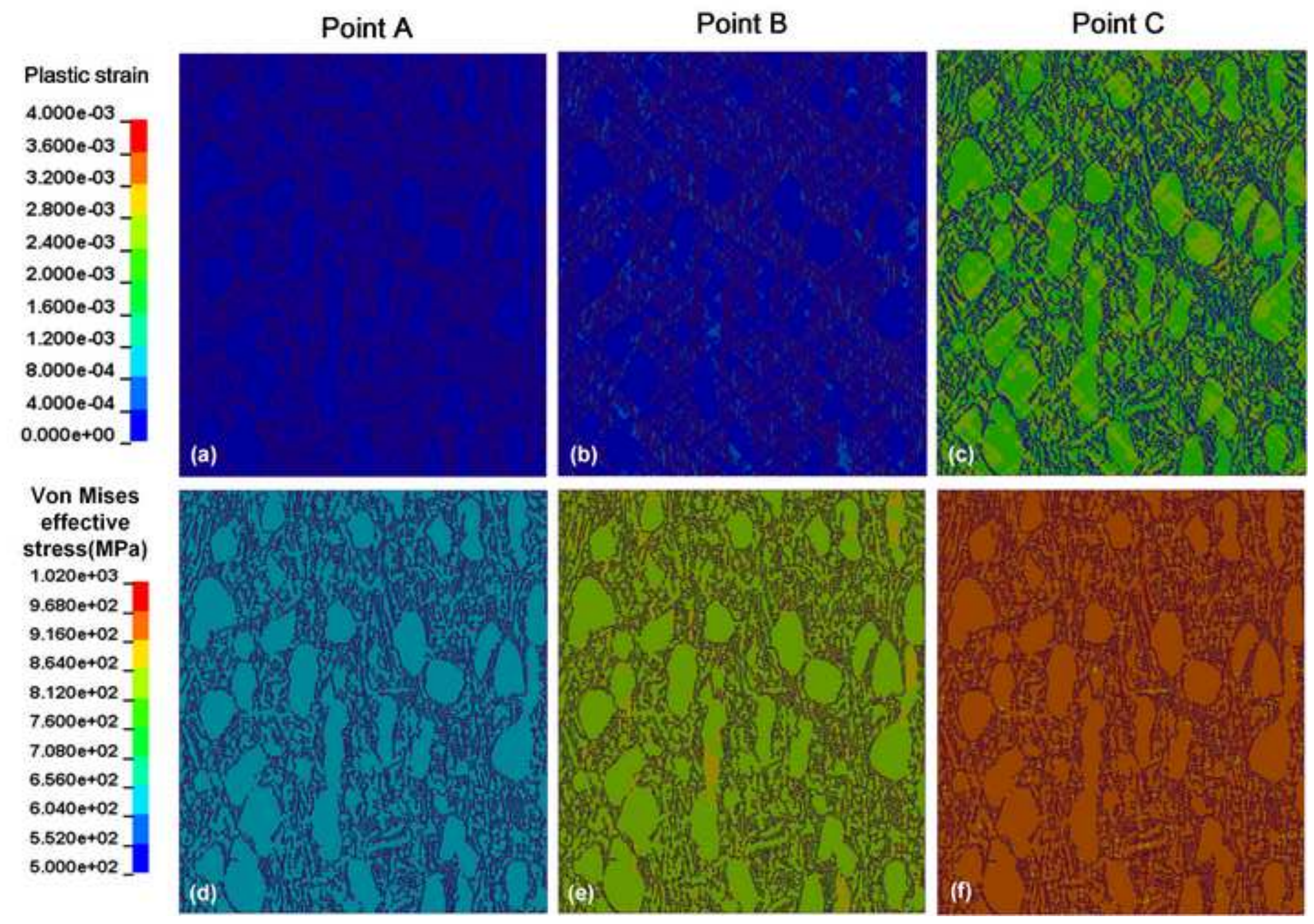




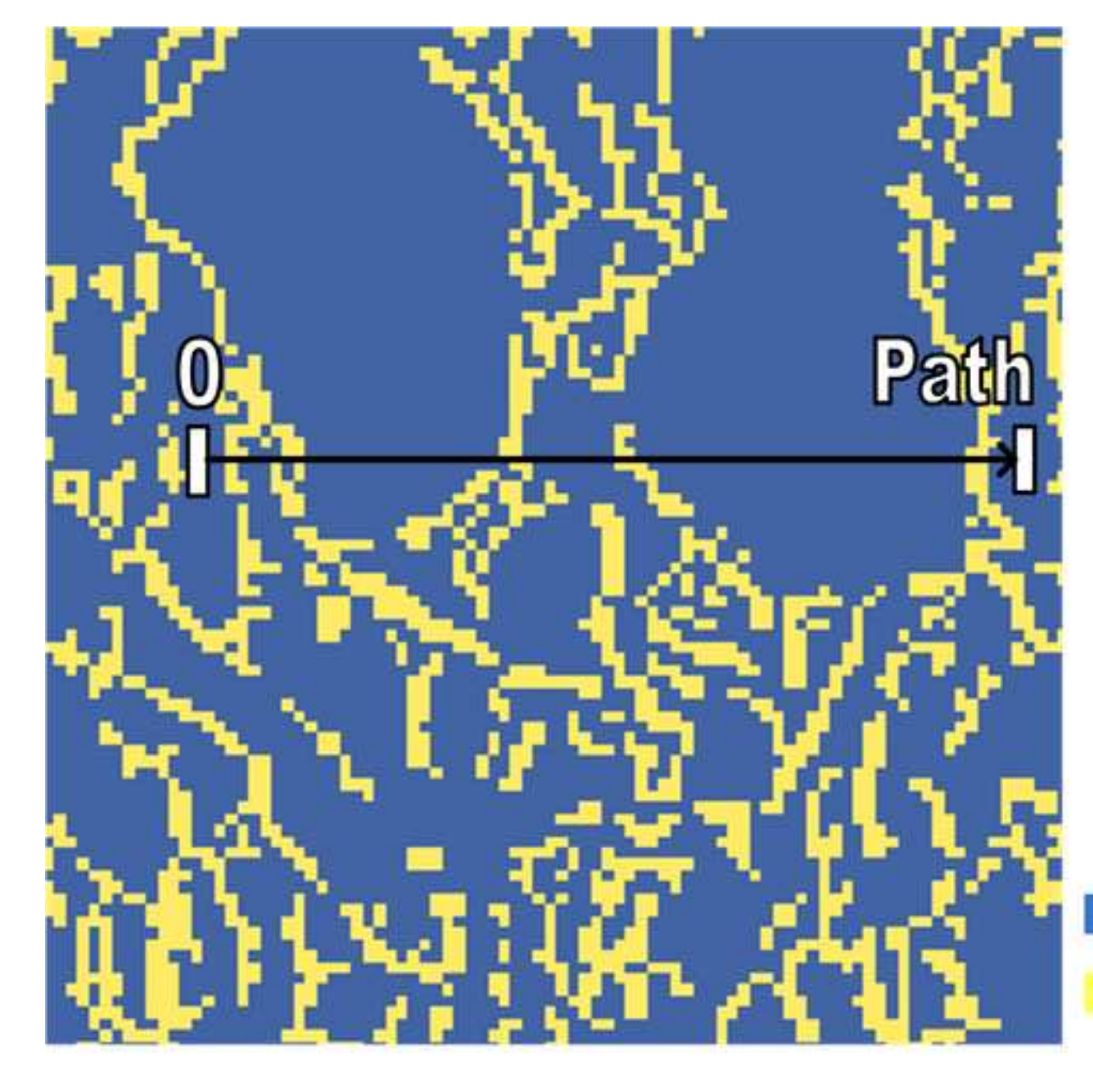

\section{$\alpha$ phase $\beta$ phase}

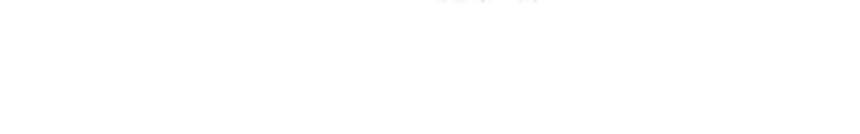



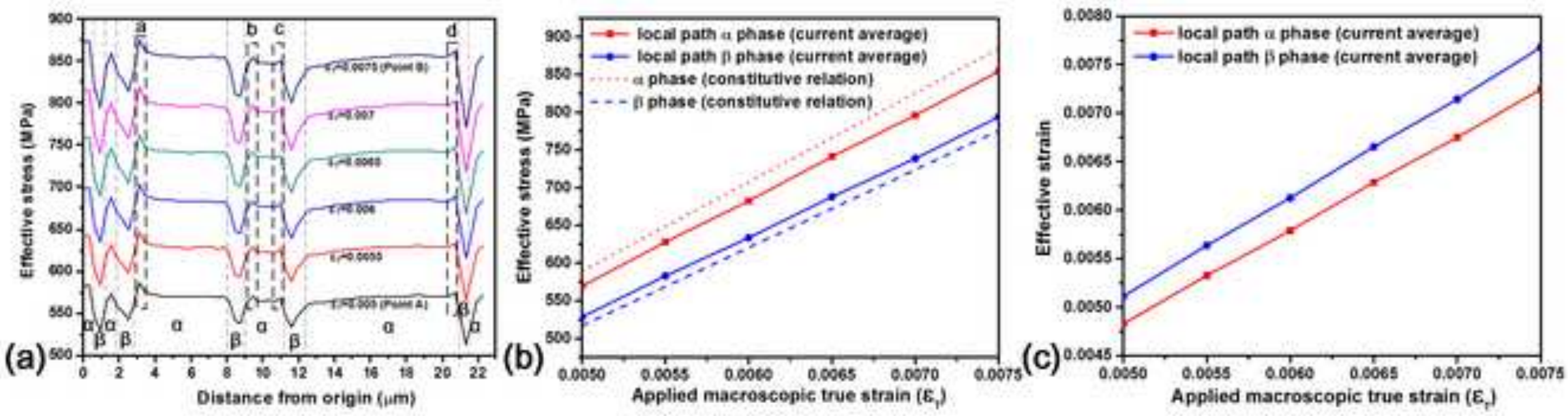

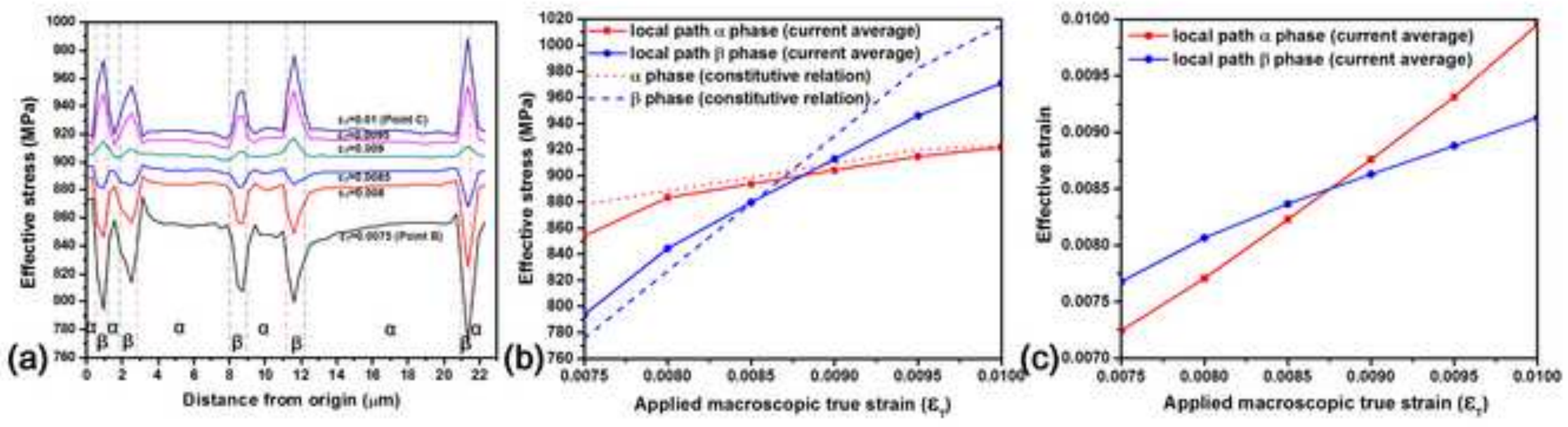

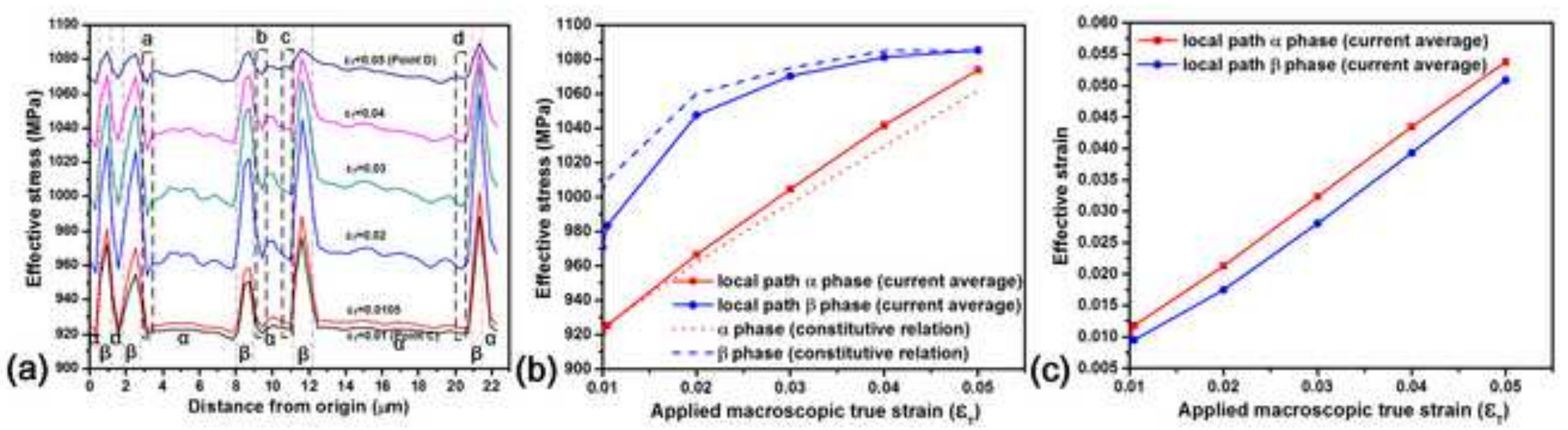


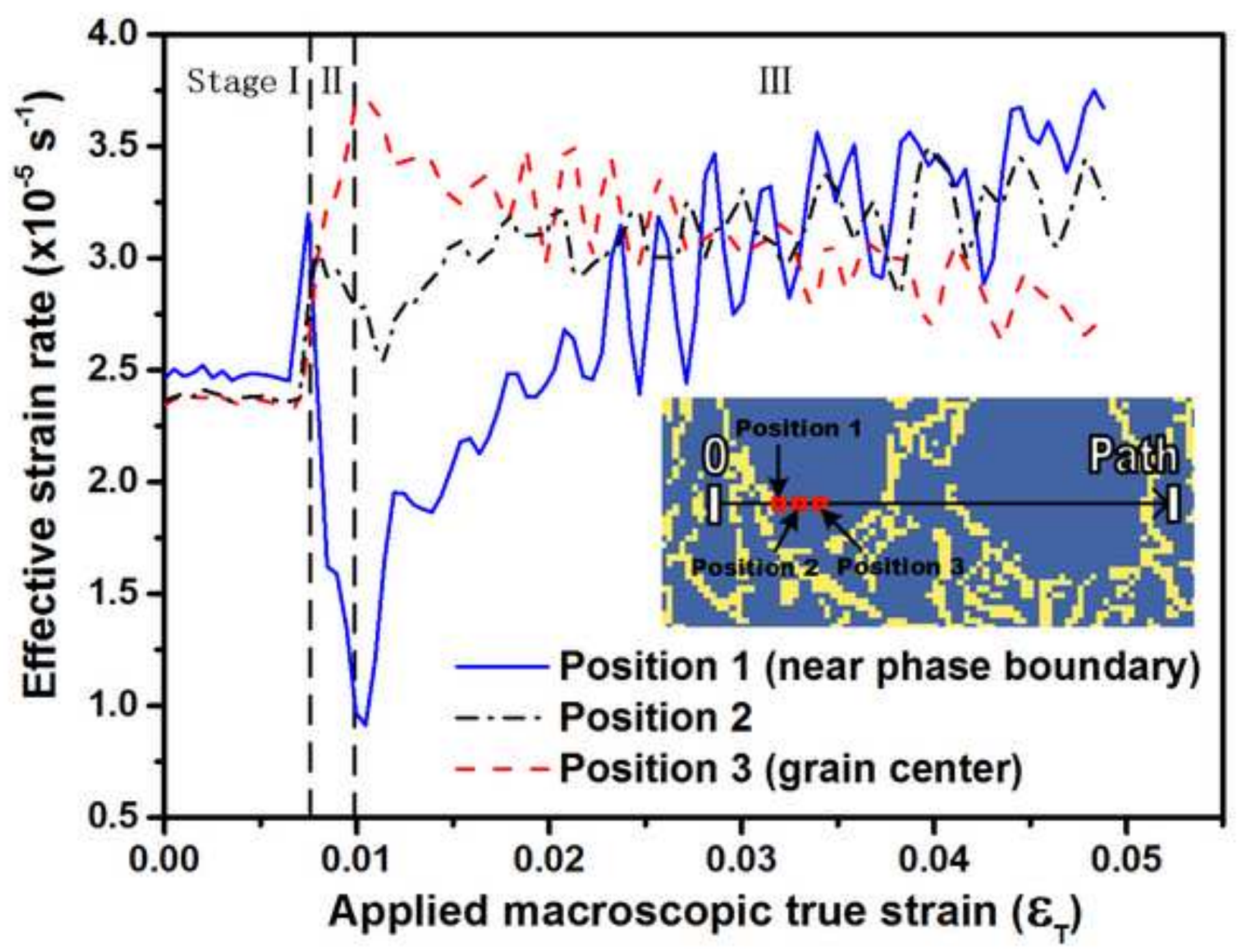


